\title{
Estresse e Coping de Árbitros de Futebol no Teste Físico FIFA
}

\author{
Alessandra Carla Peixoto Monteiro \\ Mariana Verdolin Guilherme Froeseler \\ Federação Mineira de Futebol, MG, Brasil. \\ Universidade Federal de Minas Gerais, MG, Brasil. \\ Marcela Mansur-Alves \\ Universidade Federal de Minas Gerais, MG, Brasil.
}

\begin{abstract}
Resumo:Árbitros de futebol parecem sofrer o impacto do estresse em sua atuação, especialmente em situações definidoras de seu sucesso profissional, como a Avaliação Física FIFA, que determina a aptidão física para atuação em campeonatos estaduais e nacionais. Estratégias de coping determinam a percepção de situações estressoras, e consequentemente, a forma de enfrentamento das mesmas. Este estudo teve por objetivo avaliar o impacto do teste físico FIFA nos níveis de estresse e coping de árbitros de futebol atuantes em campeonatos estaduais e nacionais (Federação Mineira de Futebol - FMF e Confederação Brasileira de Futebol - CBF, respectivamente). Responderam a Escala de Estresse Percebido, que avalia estresse negativo (distress) e coping, 42 árbitros da FMF e 17 da CBF, antes e após o teste físico FIFA. Na avaliação pré-teste, verificou-se que os árbitros da CBF apresentaram histórico de mais testes físicos, treinos por semana, anos de experiência, além de escores mais altos em coping $(\mathrm{p}<0,05)$. Quando controladas as diferenças iniciais, árbitros CBF e FMF não diferiram significativamente em coping e distress no pós-teste físico. Diante da confirmação parcial das hipóteses, sugere-se a condução de novas pesquisas na área que incluam a avaliação do tipo de estratégia de coping utilizado e características de personalidade dos árbitros.
\end{abstract}

Palavras-chave: Árbitros de Futebol, Estresse, Coping, Avaliação física, Desempenho Esportivo.

\section{Stress and Coping of Soccer Referees in the FIFA Physical Test}

\begin{abstract}
Soccer referees seem to feel the impact of stress in their performance, especially in situations that define their success at soccer, such as the FIFA Fitness Test, which determines the physical aptitude of the professional to perform in state and national tournaments. Coping strategies determine the perception of stressful situations and the consequent way of facing them. This study aimed to evaluate the impact of the FIFA Fitness Test on the stress and coping levels of soccer referees, which perform at the state and at the national level (FMF - Soccer Federation of Minas Gerais; and CBF - Brazilian Soccer Federation, respectively). 42 referees of FMF and 17 of CBF answered to The Perceived Stress Scale, which assess distress and coping, before and after the FIFA Test. At the pre-test, the CBF referees showed a major number of physical tests, weekly trainings and years of experience, and higher coping $(p<0,05)$. Controlling for initial differences, CBF and FMF referees were not significantly different on coping and distress after the test. Given the partial confirmation of the hypothesis, it is suggested to conduct further research in the area that includes the evaluation of the type of coping strategy used and personality characteristics.
\end{abstract}

Keywords: SoccerReferees,Stress,Coping,Physical Evaluation,Sports Performance. 


\title{
Estrés y Coping de Árbitros de Fútbol en la Prueba Física FIFA
}

\begin{abstract}
Resumen:Árbitros defútbol parecen sufrir el impacto del estrés en su rendimiento, especialmente en la definición de las situaciones de su éxito profesional, como la prueba de Evaluación Física FIFA, que determina la aptitud física para trabajar en los campeonatos estatales y nacionales. Las estrategias de coping determinan la percepción de las situaciones de estrés, y por lo tanto, la forma de enfrentarlos. Este estudio tuvo como objetivo evaluar el impacto del teste físico FIFA en los niveles de estrés y coping en árbitros de fútbol activos en campeonatos estatales y nacionales (Federación Minera de Fútbol - FMF, y la Confederación Brasileña de Fútbol - CBF, respectivamente). 42 árbitros FMF y $17 \mathrm{CBF}$ respondieron a la Escala de Estrés Percibido, que evalúa el estrés negativo (distress) y coping, antes y después de la prueba FIFA. En la evaluación previa a la prueba, se encontró que los árbitros CBF tenían una historia de más pruebas físicas, entrenamientos por semana, años de experiencia, y las puntuaciones más altas de coping $(\mathrm{p}<0,05)$. Cuando se controlan las diferencias iniciales, CBF y FMF árbitros no difirieron significativamente en el coping y distress después de la prueba física. Dada la confirmación parcial de las hipótesis iniciales, se sugiere llevar a cabo nuevas investigaciones incluyendo la evaluación del tipo de estrategia de coping utilizado y de la personalidad de los árbitros.
\end{abstract}

Palabras-clave: Árbitros de Fútbol,Estrés,Coping,Evaluación Física,Rendimiento Deportivo.

\section{Introdução}

A presença do árbitro de futebol é indispensável para que uma partida se realize, na medida em que ele é o responsável pelo controle do jogo e aplicação das regras (Boschilia, \& Marchi Júnior, 2008). Nas competições profissionais, os árbitros trabalham em equipe: cerca de $64 \%$ das decisões são tomadas em conjunto pelo árbitro central e árbitros assistentes (González Oya, \& Dosil Diáz, 2003; Helsen, \& Bultynk, 2004).

No contexto esportivo, o árbitro de futebol profissional pode ser considerado um atleta de alto rendimento, uma vez que necessita passar por todo um processo de treinamento físico, técnico e mental e a sua permanência nesta atividade é consignada ao seu bom desempenho esportivo (Gimeno, Buceta, Lahoz, \& Sanz, 1998; González Oya, \& Dosil Diáz,2003; Patiño, \& Cañadas, 2015; Quinteiro, 2007; Reilly, \& Warren, 2006; Velho, \& Fialho, 2015). Outro aspecto que fundamenta esta perspectiva é a presença dos quatro pilares básicos do desempenho esportivo na modalidade “arbitragem", a saber: o físico, o técnico, o tático e o psicológico (Patiño, \& Cañadas, 2015; Quinteiro, 2007; Velho, \& Fialho, 2015).

O desempenho físico diz da capacidade para se movimentar em campo acompanhando as jogadas, incorporando alternância na direção e velocidade dos deslocamentos, com agilidade, resistência e explosão.
O desempenho técnico configura-se como a capacidade para compreender, dominar e aplicar as regras do jogo, além do correto posicionamento e deslocamento em campo. O desempenho tático é representado pelo plano de trabalho elaborado antes de cada partida pela equipe de arbitragem, no qual são discutidas as diretrizes a serem tomadas dentro de campo e que estão relacionadas a integração entre os membros da equipe, as equipes de futebol escaladas, a história do jogo e do campeonato. Por fim, o desempenho psicológico envolve os aspectos mentais indispensáveis para auxiliar o árbitro a atuar de forma isenta e com equidade de critérios, pode-se ressaltar estabilidade emocional, uma leitura de jogo clara e imparcial, velocidade na tomada de decisão e estratégias de coping eficientes (González-Oya, \& Dosil Diáz, 2003; Helsen, \& Bultynk, 2004; Patiño, \& Cañadas, 2015; Quinteiro, 2007; Velho, \& Fialho, 2015).

Arbitrar é uma tarefa altamente complexa, não somente pelo fato de o árbitro ter que tomar decisões em um curto período de tempo, mas também por ficar exposto a críticas e pressões dos torcedores, atletas, técnicos e meios de comunicação (González-Oya, 2006). Esses profissionais são vítimas de abusos verbais, ameaças e, até mesmo, agressões físicas e, ao mesmo tempo, os reforçadores positivos são praticamente inexistentes (Ferreira, 2012; Ferreira, \& Brandão, 2012). Os aspectos elencados contribuem para 
que esta experiência seja exaustiva tanto emocional, quanto física e intelectualmente, constituindo, assim, possíveis fatores de estresse (Brandão, Serpa, Krebs, Araújo, \& Machado, 2011; Ferreira, \& Brandão, 2012).

Árbitros de futebol estão expostos a eventos potencialmente estressores antes, durante e depois de sua atuação (Ferreira, \& Brandão, 2012), o que, segundo De Rose Júnior (2002), constitui um fator impactante comprometedor do desempenho esportivo. Kerdijk, Kamp e Polman (2016) afirmam que as competições esportivas por si só apresentam estressores diversos, como a dor, o medo, a falta de confiança, as lesões, as relações de animosidade, além das exigências da prática desportiva e não lidar bem com elas é um fator significativo de fracasso.

A necessidade de uma rotina de preparação física, que implica em gastos financeiros e sobrecarga de atividades, também pode ser compreendida como possível fonte de estresse. Conforme Costa et al. (2010), os árbitros brasileiros percorrem, em uma partida de futebol, distâncias superiores a nove quilômetros dentro de uma área de $8.250 \mathrm{~m}^{2}$ e realizam, em média, 1.268 atividades diferentes nos 90 minutos de jogo, sendo que a cada quatro a seis segundos muda sua ação motora. Tensões ligamentares e entorses musculares estão entre as lesões mais frequentes dos árbitros de futebol, as quais podem ser adquiridas durante os treinamentos, na atuação em campo e mesmo nas avaliações físicas conduzidas bianualmente (Paes, Fernandez, \& Silva, 2011).

Dentre as situações que fazem parte da realidade dos árbitros que podem atuar como fontes de estresse está o teste físico da Fédération Internacionale de Football Association (FIFA). A federação internacional sugere à Confederação Brasileira de Futebol (CBF) a realização de testes físicos como etapa do credenciamento dos árbitros para participarem das competições. A CBF, por sua vez, recomenda às federações estaduais o mesmo procedimento, com o objetivo de controlar e selecionar os árbitros que estiverem mais bem preparados fisicamente. Este teste é realizado pelo menos duas vezes ao ano, dependendo da categoria em que o árbitro se encontra e, junto com uma avaliação teórica, ele é credenciado para a atuação anual. Diante do caráter eliminatório do teste físico, verifica-se, entre a classe, que ele se apresenta como situação potencialmente estressante para árbitros que atuam tanto a nível nacional quanto estadual.

Ainda sobre os fatores presentes no contexto da arbitragem que apresentam potencial estressor, encontra-se a questão da situação trabalhista legal da categoria. Embora o Projeto de Lei n ${ }^{\circ} 6405 / 02$, aprovado em 2013, tenha regulamentado a profissão de árbitro de futebol no Brasil, o trabalho continua sem qualquer vínculo empregatício e os árbitros são remunerados como autônomos, ou seja, as entidades não têm quaisquer responsabilidades trabalhistas, securitárias e previdenciárias ${ }^{1}$. Esta realidade implica na necessidade de manutenção de outra fonte de renda e consequente dificuldade em conciliar as atividades regularmente, devido às viagens e programas de aperfeiçoamento. Outra situação de pressão é a escala semanal para as competições. A demanda dos campeonatos não abrange todo o contingente de árbitros, o que pode significar, dependendo da percepção individual, uma situação estressora.

Empiricamente, verifica-se o número reduzido de estudos de avaliação do estresse em árbitros de futebol. Costa et al. (2010) objetivaram identificar os principais fatores causadores de estresse em 102árbitros federados em Minas Gerais. Os participantes preencheram o Teste de Estresse Para Árbitros (TEPA), que se divide em três dimensões: biológica, psicológica e social. O estresse biológico é compreendido por modificações do estado fisiológico que podem, inclusive, interferir em seu desempenho, tais como: cansaço físico, sono, fome, dor, e disfunções orgânicas. $\mathrm{O}$ estresse social diz respeito a relações sociais em todos os ambientes frequentados pelo indivíduo, e que podem influenciar em seu rendimento. $O$ estresse psicológico tem como característica a preocupação com o cumprimento da função de árbitro enquanto disciplinador e controlador das regras do jogo.

Os resultados obtidos por Costa et al. (2010) indicaram que os itens relativos à dimensão psicológica e social obtiveram as maiores pontuações médias, com destaque para: não poder cumprir uma escala (dimensão psicológica), locais com falta de segurança, trajetos de ida e vinda inseguros, falta de valorização profissional e descompromisso da equipe de trabalho (dimensão social). Os autores concluíram que, apesar

${ }^{1}$ Informações retiradas do Projeto de Lei No 6.405, de 2012, do Senado Federal, que Regula a profissão de árbitro de futebol. Texto disponível em Http://imagem.camara.gov.br/Imagem/d/pdf/DCD11ABR2002.pdf\#page=143 
dos fatores sociais e psicológicos serem destacados como mais estressantes pelos árbitros, deve-se considerar a influência recíproca da tridimensionalidade (social, psicológica e biológica) dos fatores que estimulam o surgimento do estresse nessa população.

Claudino et al. (2012) utilizaram o mesmo instrumento que Costa et al. (2010), o TEPA (Silva, 2004), para avaliar a percepção de estresse de 30 árbitros amadores atuantes em Minas Gerais. Os resultados apontaram para diferenças quanto às fontes de estresse percebido entre árbitros mais e menos experientes. Os árbitros mais experientes citaram como fatores mais estressantes: "não saber com quem vai apitar" e "atraso das equipes para iniciar a partida". Enquanto isso, os menos experientes apontaram como maior estressor "não ter reconhecimento e/ou valorização". Concluiu-se que somente os fatores ligados a estressores sociais, e não físicos, foram capazes de diferenciar os dois grupos (Claudino et al., 2012). Apesar de terem encontrado tais resultados, os autores não conduziram análises de comparação com os escores totais obtidos pelos árbitros nos testes de estresse, o que impossibilitou estabelecer relações entre quantidade de estresse percebido e experiência na arbitragem.

Pereira, Santos e Cillo (2007) afirmam que o estresse está presente quando a quantidade de exigências e de tensões é excessiva para o indivíduo, testando sua resistência às pressões e suas habilidades para lidar com demandas e mudanças do ambiente. Segundo Ribeiro e Marques (2009), o estresse pode ser definido e avaliado segundo três perspectivas: foco na consequência, foco na causa e foco no processo. O primeiro diz respeito às alterações físicas e mentais apresentadas quando o indivíduo se defronta com estímulos ameaçadores. O segundo e o terceiro se diferenciam na medida em que o estresse com foco na causa relaciona-se a uma avaliação da situação em si (também chamada de abordagem ambiental ou industrial), enquanto que no estresse com foco no processo considera-se a avaliação feita pelo indivíduo (estresse percebido), que também é denominado de abordagem psicológica.

Dentre os teóricos que adotam a perspectiva psicológica está Lazarus (1964), que afirma que o estresse é fruto da interação entre o meio ambiente e o indivíduo, mais especificamente, de quando o indivíduo avalia que as demandas (externas e internas) ultrapassam sua capacidade de manejo. De acordo com Krohne (2001), esse autor é representante de uma abordagem do estresse psicológico inserida no campo da PsicologiaCognitiva, contrapondo-se a perspectivas baseadas na fisiologia e psicobiologia do estresse. Destaca-se a importância desta perspectiva, que não direciona a um elemento estressor ou tipologias de situações, mas ao quanto o indivíduo avaliou como estressora uma situação qualquer, real ou imaginária, para a qual atribuiu um significado particular (Cohen, Kamarck, \& Mermelstein, 1983).

Uma habilidade fundamental e determinante para o bom desempenho esportivo do árbitro de futebol é a forma como lida com tantos estímulos estressores. Para lidar com o estresse, Gomes, Coimbra, Guillén, Miranda e Bara Filho (2007) defendem a utilização de estratégias de coping que podem ser entendidas como formas de enfrentamento ao estresse. Segundo Folkman e Lazarus, as estratégias de coping são ações deliberadas que podem ser aprendidas, usadas e descartadas (Antoniazzi, Dell'Anglio, \& Bandeira,1998), podendo ser divididas em duas categorias funcionais: as focadas na emoção e as focadas no problema (Lazarus, \& Folkman, 1984). Na primeira, o indivíduo utiliza-se de estratégias que visam à diminuição da perturbação emocional, sendo mais adaptável quando os estressores são em grande parte incontroláveis. As estratégias compreendidas na segunda categoria têm por objetivo gerir o problema que se encontra na gênese da perturbação do sujeito, buscando controlar ou alterar a situação que originou o estresse (Nicholls, Holt, Polman, \& Bloomfield,2006). De uma forma geral, essas estratégias se mostram mais adequadas a situações de ameaça nas quais há risco iminente risco, mas que ainda são reversíveis (Lazarus, \& Folkman, 1984).

A literatura científica sobre coping apresenta três gerações de pesquisadores com filiações epistemológicas bem distintas (Antoniazzi et al., 1998). A primeira, desde o início do século XX, traz pesquisadores vinculados à Psicologia do Ego, como Menninger, Haan e Vaillant, que concebem o coping enquanto correlato aos mecanismos de defesa, motivado interna e inconscientemente como forma de lidar com conflitos sexuais e agressivos (Vaillant, 1994). Segundo Carver e Connor-Smith (2010), a segunda abordagem acerca de coping é a mais conhecida e corresponde à perspectiva de Folkman e Lazarus, que o entendem como um processo de interação indivíduo - ambiente, que representa esforços cognitivos e comportamentais que visam à redução de um mal-estar provocado por demandas internas e externas de uma situação. Uma 
abordagem mais recente propõe o coping como ação regulatória, em uma perspectiva desenvolvimentista (Skinner, 1999). Chamada de Teoria Motivacional do Coping (Ramos, Enumo, \& Paula, 2015), ela engloba os esforços individuais para manter, restaurar ou reparar as necessidades psicológicas básicas de: relacionamento, competência e autonomia.

Desde o início da década de 1990, pesquisadores do esporte começaram a se interessar pelo estudo do coping no contexto esportivo (Gould, Ecklund, \& Jackson,1993; Nicholls, Holt, Polman, \& Bloomfield, 2006). A incapacidade de lidar com o estresse é um fator significativo no fracasso do desenvolvimento da performance de forma plena nas competições esportivas (Lazarus, 2000). Hardy, Jones e Gould (1996) defendiam que as estratégias de coping seriam um fator essencial para explicar as diferenças de níveis de desempenho esportivo, apesar disso os artigos sobre o assunto ainda eram pouco frequentes.

A fim de avaliar a relação entre diferentes estratégias de coping e desempenho esportivo (percebido e avaliado objetivamente), Gaudreau, Nicholls e Levy (2010) realizaram um estudo com 54 jogadores de golfe, do sexo masculino, participantes de uma competição desta modalidade, ocorrida no Reino Unido. Foram analisadas seis rodadas consecutivas, sendo que inicialmente os participantes responderam a um questionário sociodemográfico. Após três horas de cada partida, eles respondiam a questionário individual avaliando seu desempenho na partida, ao Inventário de Coping de Desporto de Competição (CICS; Gaudreau, \& Blondin, 2002) e ao Inventário de Estresse (Kowalski, \& Crocker, 2001). Entre os resultados, os autores encontraram correlações positivas e significativas entre o estresse percebido e o uso de estratégias de coping de evitação ou distração, que também estavam relacionadas ao pior desempenho. Enquanto isso, estratégias de coping orientadas para a tarefa apresentaram correlações significativas e positivas com desempenho percebido.

A partir da revisão de literatura apresentada, constata-se que a arbitragem é permeada por fatores estressantes que demandam dos profissionais a utilização de diferentes estratégias de enfrentamento. Assim, o presente estudo visa contribuir para a compreensão do estresse e das estratégias de coping no desempenho dos árbitros de futebol, possibilitando a construção de conhecimentos que poderão auxiliar o profissional da Psicologia que trabalha com esporte a desenvolver estratégias de trabalho e preparação dos árbitros mais adequados, visando aumentar o número de credenciamentos para o ano de trabalho. Ademais, esse trabalho justifica-se pela lacuna existente na literatura de estudos que avaliem essa população.

Nesse sentido, o presente estudo objetiva investigar o impacto do teste físico FIFA nos níveis de estresse e coping percebidos por árbitros do futebol mineiro, comparando dois momentos (antes e depois do teste), e considerando variáveis como idade, experiência e nível de atuação como possíveis moderadores desse impacto. Tem-se como hipótese inicial que o teste físico acarretará em aumento na percepção do estresse, sendo que os árbitros mais experientes (atuantes em campeonatos nacionais) deverão ser menos afetados do que os menos experientes (atuantes no campeonato estadual).

\section{Método}

\section{Delineamento}

O estudo é de cunho quantitativo, descritivo e exploratório. Estudos que contemplam a análise quantitativa geralmente trabalham com coleta de dados e classificação de informações utilizando de estatísticas apropriadas no intuito de evitar possíveis distorções de análise e interpretação, o que confere uma maior margem de segurança. Um estudo quantitativo pode usar de análise descritiva para traduzir seus resultados, ou seja, ele levanta os dados e o porquê destes dados. A justificativa para se adotar a análise exploratória é a escassez de informações sobre o estresse provocado pelo teste físico da FIFA em árbitros profissionais do futebol (Dalfovo, Lana, \& Silveira, 2008).

\section{Participantes}

Foram convidados a participar deste estudo 59 árbitros e assistentes profissionais, todos do sexo masculino, do estado de Minas Gerais. A média de idade dos árbitros foi de 32,73 anos (DP=5,86 anos) e a escolaridade variou de ensino médio completo $(22 \%$ da amostra) a pós-graduação ( $16,9 \%$ da amostra), sendo que a maioria deles possuía ensino superior completo (61\%). O tempo médio de experiência na arbitragem foi de 10,15 anos (DP= 5,33 anos). Nos procedimentos de análise dos dados os participantes foram ainda subdivididos segundo seu nível de atuação na arbitragem: se regional ( $\mathrm{n}=42$, Federação Mineira de Futebol -FMF) ou nacional ( $n=15$, Confederação Brasileira de Futebol - CBF). 


\section{Instrumento}

Foi utilizada a Escala de Estresse Percebido, desenvolvida nos Estados Unidos por Cohen et al. (1983), e destinada à avaliação do modo como a pessoa se percebe em situações possivelmente estressoras que o meio ambiente proporciona. A versão brasileira foi desenvolvida por Luft, Sanches, Mazo e Andrade (2007), apresentando índices satisfatórios de consistência interna e validade de construto. A fundamentação teórica desta escala é a teoria de Lazarus (1964), que preconiza serem eventos estressores aqueles que excedem os recursos internos percebidos pelo indivíduo para lidar com as situações que the parecem ameaçadoras. Nessa perspectiva de estresse, o foco encontra-se na avaliação cognitiva feita pelo indivíduo sobre o evento.

A Escala de Estresse Percebido possui 14 questões com opções de resposta que variam de nunca a sempre. As questões com conotação positiva $(4,5,6$, 7, 9, 10 e 13) devem ser invertidas antes de somadas, enquanto as demais (negativas) são somadas diretamente. O total da escala é a soma das pontuações das 14 questões, tendo a pontuação máxima de 56 pontos. Os resultados também podem ser analisados segundo a categorização: percepção elevada de stress $(\geq 75 \%)$ e percepção stress moderada $(<75 \%)$.

A interpretação do resultado da Escala de Estresse Percebido trabalha com dois níveis de informação, o distresse e o coping. O primeiro esclarece como o indivíduo percebe as situações como agentes estressores, enquanto o segundo avalia sua interpretação acerca de sua capacidade para resolvê-las, ou seja, como consegue lidar com estas situações, e como entende suas estratégias de enfrentamento para situações estressantes. A escala e suas várias versões já foram validadas em diferentes países, tais como: Portugal (Pais-Ribeiro, \& Marques, 2009), Japão (Mimura, \& Griffiths, 2004; 2008), Turquia (Örücü, \& Demir, 2008), Brasil (Luft et al., 2007; Reis, Hino, \& Añez, 2010), Espanha (Remor, 2006), México (Ramírez, \& Hernández, 2007) e Dinamarca (Olsen, Mortensen, \& Bech, 2004), apresentando boas propriedades psicométricas. Ademais, por não possuir questões relacionadas a contextos específicos, torna-se adequada para uso em diferentes gêneros e grupos etários.

\section{Procedimentos}

Antes de seu início, a pesquisa foi submetida à apreciação e aprovada pelo Comitê de Ética em Pesquisa da Universidade Federal de Minas Gerais (COEP-UFMG) (CAAE $n^{\circ}$ 53351216.4.0000.5149). A condução da pesquisa se deu em duas etapas, pois foi avaliado um grupo em cada dia. O primeiro grupo avaliado foram os árbitros e assistentes CBF e, uma semana após esta coleta, o segundo grupo, dos árbitros e assistentes da FMF, passou pelo mesmo processo. Os testes foram realizados à noite, na pista de corrida do Centro de Treinamento Esportivo da UFMG. Os participantes já haviam sido instruídos a respeito dos procedimentos da pesquisa e antes de iniciarem o teste físico, eles leram e assinaram o Termo de Consentimento Livre e Esclarecido (TCLE) e preencheram a primeira Escala de Estresse Percebido. Em seguida, realizaram o teste físico.

O teste físico FIFA consiste em uma prova de corrida com seis tiros curtos de 40 metros, para avaliar a velocidade que o indivíduo atinge, e outra prova de 20 tiros de 150 metros, com mais 50 metros de recuperação em caminhada, para avaliar a resistência. Os critérios de tempo deste teste são diferenciados por função (árbitro e assistente), sexo (masculino e feminino) e categoria (FIFA, CBF e Federações). Os tempos para realização de cada prova são: Tiro Curto: máximo de 6,4 segundos para árbitros e 6,2 segundos para assistentes; Tiro Longo: árbitros - corrida de 150 metros em até 30 segundose recuperação de 50 metros em 40 segundos; assistentes - corrida de 150 metros em até 30 segundos e recuperação de 50 metros em 45 segundos².

Após realizarem o teste físico descrito acima, foi dado um tempo para participantes da pesquisa se recuperarem, só então foi aplicada a segunda Escala de Estresse Percebido. Em ambos os momentos (antes e após o teste físico), as aplicações ocorreram de forma coletiva e tiveram duração aproximada de 30 minutos cada.

\section{Análise dos dados}

Verificou-se, por meio do teste de KolmogorovSmirnov, que os dados não seguem distribuição normal ( $>0,005)$, levando à escolha pela utilização de testes estatísticos não paramétricos. Inicialmente foram conduzidas análises descritivas dos dados relativos à atuação participantes na arbitragem (experiência, número de testes FIFA realizados e número de reprovações, frequência semanal de treinos), além das informações sobre idade e os escores obtidos na Escala de Estresse Percebido (distress e coping) antes do teste físico. Essas mesmas vari-

${ }^{2}$ Informações sobre o teste FIFA foram retiradas de: https://refnews.wordpress.com/2012/06/27/fifa-muda-teste-fisico-para-arbitros/. 
áveis foram submetidas a análises de comparação entre grupos (teste Mann-Whitney para amostras independentes) a fim de verificar diferenças entre os árbitros CBF e FMF, além de análises de correlação para estabelecer o grau de associação entre as variáveis.

A fim de responder à hipótese central do trabalho (impacto do teste físico FIFA no estresse percebido pelos árbitros), empreendeu-se uma análise de covariância (Ancova), na qual as variáveis que apresentaram diferenças no teste de Mann-Whitney entraram como covariáveis, os escores de distress e coping no pós-teste como variáveis dependentes e os níveis de atuação como fatores independentes. Esse tipo de análise busca garantir que as diferenças encontradas no pós-teste não sejam explicadas por diferenças anteriores ao teste físico.

\section{Resultados}

\section{Impacto do teste físico FIFA no estresse e coping de árbitros mineiros}

A Tabela 1 apresenta os resultados de análises descritivas conduzidas com as variáveis idade, anos de experiência na arbitragem, quantidade de vezes que os árbitros se submeteram ao teste físico FIFA, quantidade de reprovações, número de treinos por semana. Nessas análises os árbitros foram divididos por nível de atuação (regional somente ou nacional) (Tabela 1). Em seguida, avaliou-se se o nível de atuação do árbitro (regional ou nacional) seria moderador da percepção do impacto do teste FIFA no estresse e coping, partindo-se do pressuposto teórico de que os árbitros nacionais (CBF), por terem mais experiência e já terem passado pelo teste físico maior número de vezes do que os árbitros que apitam apenas campeonatos estaduais (FMF), sofreriam menos impacto dele.

A fim de verificar se os grupos de árbitros (FMF e CBF) possuem diferenças em sua caracterização geral no tempo 1 (antes da realização do teste físico FIFA), foram realizados testes estatísticos de comparação entre grupos. O teste Mann-Whitney para amostras independentes apontou que os árbitros FMF e CBF não diferem com relação à idade $(\mathrm{p}=0,343)$ e ao número de reprovações no teste FIFA ( $\mathrm{p}=0,408$ ). Contudo, os árbitros que apitam a nível nacional diferem significativamente daqueles que apitam a nível regional no que se refere ao número de testes físicos realizados, ao número de vezes que treina por semana e aos anos de experiência em arbitragem $(p<0,05)$, sendo que os primeiros realizaram o teste FIFA mais vezes, treinam mais vezes por semana e são, por sua vez, mais experientes do que os segundos.

Também foi avaliado se os dois grupos de árbitros possuíam escores semelhantes em distress e coping antes do teste físico (pré-teste). Os dois grupos não diferiram significativamente nos escores em distress no pré-teste $(\mathrm{p}=0,798)$, porém os escores em coping foram significativamente mais altos para o grupo de árbitros CBF $(\mathrm{p}=0)$. A Figura apresenta as médias dos dois grupos em distress e coping antes e depois do teste físico FIFA.

Tabela 1

Estatísticas descritivas (média e desvio-padrão) das principais variáveis que caracterizam a amostra, segundo nível de atuação.

\begin{tabular}{lcccc}
\hline & & $\mathrm{N}$ & Média & Desvio-padrão \\
\hline \multirow{2}{*}{ Experiência (em anos) } & FMF & 42 & 8,29 & 4,46 \\
\multirow{2}{*}{ Quantidade de testes FIFA realizados } & CBF & 15 & 14,33 & 4,64 \\
& FMF & 42 & 6,46 & 5,01 \\
Quantidade de reprovação no teste FIFA & CBF & 15 & 15,40 & 11,26 \\
& FMF & 42 & 0,86 & 1,54 \\
Idade & CBF & 15 & 0,53 & 1,3 \\
\multirow{2}{*}{ Número de treinos por semana } & FMF & 42 & 32,07 & 6,29 \\
& CBF & 15 & 34,40 & 4,64 \\
& FMF & 42 & 2,98 & 1,24 \\
\hline
\end{tabular}

FMF: Federação Mineira de Futebol; CBF: Confederação Brasileira de Futebol. 
$\mathrm{Na}$ sequência, objetivou-se verificar qual o impacto do teste físico nos escores de ambos os grupos, comparando antes e depois da realização do mesmo. A Tabela 2 apresenta as médias dos dois grupos antes e depois do teste FIFA para a subescalas de distress e coping.

Como os dois grupos diferiram significativamente nos escores de coping do pré-teste, ou seja, eles já eram diferentes antes da realização do teste físico FIFA, é necessário controlar essa diferença inicial a fim de verificar se a mesma se mantém ou não (aumento ou diminui após a realização do teste físico). Para tanto, utilizou-se a Ancova, na qual os escores de coping do pré-teste entraram como covariáveis, bem como a experiência a arbitragem e o número de testes físicos realizados (que também deferiram entre os grupos, conforme apresentado acima), os escores de coping no pós-teste como variáveis dependentes e os níveis de atuação como fatores independentes.

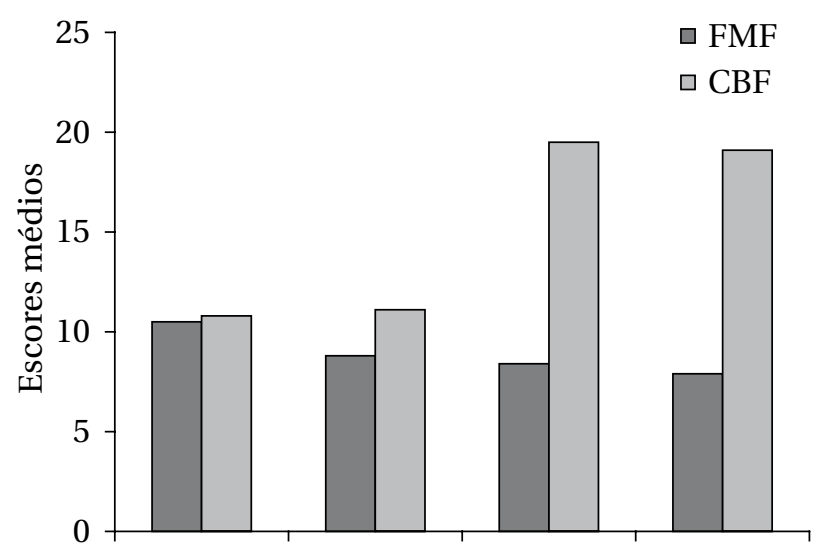

Figura

Escores médios de distress e coping antes e depois do teste físico, por nível de atuação (FMF e CBF).

Tabela 2

Média e desvio-padrão para as subescalas de distress e coping de acordo com o nível de atuação.

\begin{tabular}{lcccc}
\hline & \multicolumn{2}{c}{ Média } & \multicolumn{2}{c}{ Desvio-padrão } \\
\cline { 2 - 5 } & FMF & CBF & FMF & CBF \\
\hline Distress antes & 10,50 & 10,76 & 4,55 & 3,36 \\
Distress depois & 8,81 & 11,12 & 4,27 & 3,98 \\
Coping antes & 8,40 & 19,12 & 4,95 & 4,57 \\
Coping depois & 7,86 & 19,06 & 5,07 & 4,44 \\
\hline
\end{tabular}

FMF: Federação Mineira de Futebol $(n=42)$;CBF:

Confederação Brasileira de Futebol $(\mathrm{n}=17)$.
Os resultados encontrados apontam que, quando controlada a diferença de escores em coping observados no pré-teste, a experiência e o número de testes físicos realizados, os árbitros CBF e FMF não diferem nos seus escores em coping após a realização do teste físico FIFA ( $\mathrm{Z}=2,987 ; \mathrm{p}=0,09)$.

Já com relação às diferenças entre os árbitros nos escores de distress após a realização do teste FIFA, apenas as variáveis experiência e quantidade de testes já realizados entraram nas análises de covariância como covariáveis, já que os dois grupos não diferiram significativamente nos níveis de distress no pré-teste (conforme pode ser observado na Figura). Os resultados encontrados também apontam que não há diferenças estatisticamente significativas entre árbitros CBF e FMF nos níveis de distress após o teste físico FIFA, quando controladas as variáveis experiência e número de testes físicos já realizados $(\mathrm{Z}=1,067 ; \mathrm{p}=0,306)$.

\section{Associação entre distress, coping e experiência na arbitragem}

Por fim, foram empreendidas análises de correlação de Spearman entre as variáveis de interesse do estudo, considerando a amostra total $(n=59)$. A idade dos participantes se correlacionou de forma positiva e significativa com a quantidade de testes físicos já realizados pelo árbitro $(\mathrm{r}=0,653 ; \mathrm{p}=0)$ e com a experiência, medida pelo número de anos de atuação $(\mathrm{r}=0,654 ; \mathrm{p}=0)$.

Com relação à associação entre as variáveis idade e anos de atuação e a pontuação dos árbitros nos dois fatores da Escala de Estresse Percebido (distress e coping), não foram encontradas correlações estatisticamente significativas entre o distress e as variáveis idade, experiência e quantidade de testes FIFA já realizados. Por outro lado, a dimensão de coping percebido se correlacionou se forma positiva e significativa com o número de testes físicos FIFA já realizados pelo arbitro ( $r=0,369$; $p=0,004)$ e com a experiência na arbitragem $(\mathrm{r}=0,410 ; \mathrm{p}=0,001)$.

\section{Discussão}

O presente trabalho objetivou verificar o impacto do teste físico FIFA no estresse percebido e as estratégias de coping utilizadas por árbitros profissionais atuantes no estado de Minas Gerais. Considerou-se, para estas análises, a idade, anos de experiência na arbitragem, número de testes físicos já realizados e o nível de atuação (regional ou nacional). 
De forma geral, os resultados encontrados confirmam, apenas parcialmente, as hipóteses levantadas. Não foi encontrada associação entre número de testes físicos já realizados, experiência em anos, e o estresse percebido pelos árbitros, ao passo que essas mesmas variáveis se correlacionaram com o coping percebido. Este resultado é interessante na medida em que parece indicar que a percepção do teste FIFA como evento estressor não está relacionada à experiência do árbitro e nem ao número de vezes que já realizou o teste; ao passo que, quanto mais experiência e maior quantidade de testes FIFA já realizados pelo árbitro, maior é a sua percepção de que é capaz de lidar com eventos estressores (coping mais alto).

Os resultados encontrados se aproximam aos de Claudino et al. (2012), em estudo realizado com árbitros amadores mineiros. Os autores apontam que são os estressores sociais (falta de reconhecimento, atrasos para início das partidas e insegurança com relação à equipe de arbitragem de um jogo), e não os físicos (tais como dor, cansaço físico e fome), aqueles capazes de diferenciar árbitros mais experientes daqueles menos experientes. Resultado semelhante também foi obtido por Costa et al. (2010), apontando que as dimensões psicológicas e sociais do estresse são aquelas que parecem estar mais presentes no cotidiano dos árbitros profissionais e podem, por sua vez, impactar o seu desempenho.

Quanto à diferença no coping entre árbitros mais e menos experientes, é possível hipotetizar que a experiência em arbitragem e o envolvimento em diferentes situações estressoras ao longo dos anos pode atuar de forma positiva para a percepção do árbitro quanto à sua capacidade de lidar com os estressores, tornando-os mais aptos e confiantes. Resultado similar foi encontrado por Coimbra, Bara, Andrade e Miranda (2013) em sua pesquisa com atletas de alto rendimento e suas habilidades de coping, que encontrou correlação positiva entre coping e nível de atuação esportiva, caracterizando o nível esportivo pela quantidade e qualidade das competições enfrentadas pelos esportistas estudados, ou seja, pelo tempo de prática dos mesmos.

Analisando os resultados dos árbitros (CBF e FMF) na escala de distress, percebe-se que eles não diferiram, de forma significativa, antes e depois do teste físico. Isso pode sugerir que o teste não se apresenta como um evento estressor para nenhum dos dois grupos. Tal resultado não confirma a hipótese inicialmente levantada, de que o teste FIFA seria percebido como evento estressor (maiores pontuações antes da realização do mesmo), e de que árbitros mais experientes (CBF) perceberiam como menos estressor do que árbitros menos experientes (FMF), tal como apontado por Cerqueira, Silva e Marins (2011). Esses autores ressaltam uma inadequação do teste físico FIFA em relação às demandas físicas necessárias para a atuação do árbitro. Além de avaliar aspectos diferentes, parece que o teste é desnecessariamente mais rigoroso do que deveria, ou seja, poderia acarretar uma carga desnecessária e aumento de estresse. Além do mais, o mesmo teste pode aumentar o risco do árbitro em adquirir uma lesão física, o que comprometeria seu desempenho ao longo do ano de trabalho (Paes et al., 2011). Apesar dos últimos dados apontarem o excesso de carga do teste FIFA, deve-se atentar pelos estudos citados anteriormente que demonstraram que os fatores associados como estressores pelos árbitros não são os físicos, e sim os de cunho social e psicológico, como falta de reconhecimento, insegurança quanto à equipe de trabalho e atrasos.

Quanto ao coping percebido, os dois grupos diferiram de forma significativa antes da realização do teste físico, com o grupo CBF obtendo pontuações mais elevadas do que o FMF. Esse resultado parece estar relacionado com a experiência do árbitro (discutida anteriormente), já que árbitros CBF são mais experientes do que os que atuam somente em campeonatos estaduais. Quando se comparam os escores em coping, antes e depois do teste físico FIFA, para ambos os grupos, não há diferenças significativas entre esses dois momentos. Isso significa que os escores de coping percebido não foram afetados pela realização do teste físico FIFA.

Por fim, dentre as lacunas apresentadas no presente estudo, ressalta-se que a escala utilizada no presente estudo não avalia tipo de estratégia de coping utilizado pelo indivíduo, e sim a magnitude com que se percebe capaz de lidar com estressores. A literatura aponta que há estratégias de enfrentamento diferentes para lidar com eventos estressores, e que elas podem ser mais ou menos funcionais (Anshel, 2001; Antoniazzi et al., 1998). Seria, portanto, interessante considerar, em estudos futuros, que árbitros mais experientes podem utilizar estratégias de coping mais funcionais do que árbitros menos experientes, ao investigar o tipo de estratégia de enfrentamento utilizado por eles. 
Também é importante destacar que o delineamento utilizado no presente estudo não permite afirmar se foi a experiência em arbitragem o fator que proporcionou aumento da percepção de coping nos árbitros. Sabe-se, por exemplo, que características de personalidade se associam com a percepção do indivíduo acerca de suas competências para lidar com eventos estressores. Lopez e Santelices (2011) apontam que pessoas com Neuroticismo mais alto, ou seja, mais propensas a experimentar emoções negativas, mais ansiosas, inseguras ou vulneráveis, se percebem com menos competência para fazer face às situações estressoras. Além disso, estes mesmos indivíduos tendem a perceber situações cotidianas comuns como potencialmente estressoras, aumentando, também seu nível de estresse percebido. Nesse sentido, estudos futuros sobre estresse e coping em árbitros de futebol deveriam incluir a avaliação de fatores de personalidade como potenciais moderadores da relação entre experiência, estresse e coping (Allen, Greenlees, \& Jones, 2011; Bara, Ribeiro, \& Garcia, 2005; Malinauskas, Dumciene, Mankus, \& Vekcunas, 2014).

\section{Considerações finais}

Diante dos resultados encontrados é possível concluir que o teste físico FIFA não se constitui como um evento estressor para os árbitros avaliados, independentemente do nível de atuação. Esse resultado pode ser compreendido quando considerados estudos anteriores que encontraram fatores sociais e psi- cológicos como estressores mais relevantes para árbitros de futebol (Claudino et al., 2012; osta et al., 2010).

Foram encontradas diferenças estatisticamente significativas entre os árbitros antes do teste físico no que diz respeito ao coping percebido, experiência, número de testes físicos realizados e idade. No pós-teste, a partir do controle dessas variáveis, não foram verificadas diferenças adicionais entre os árbitros. Por fim, correlações positivas e estatisticamente significativas foram encontradas entre a dimensão de coping percebido e o número de testes físicos FIFA já realizados e a experiência na arbitragem. Não foram encontradas correlações significativas entre tais variáveis e a dimensão distress.

Dentre as limitações do presente estudo, pode-se apontar o instrumento utilizado para a avaliação do estresse (Escala de Estresse Percebido). Por ser uma escala inespecífica, ela pode ter sido incapaz de apontar se o estresse percebido está relacionado a eventos físicos, sociais ou psicológicos, sugerindo a utilização de outros instrumentos em futuras investigações.

Ratifica-se a importância do estudo no sentido de trazer novos conhecimentos acerca dos fatores que podem interferir na atuação dos árbitros brasileiros, o que proporcionaria intervenções psicológicas mais eficazes e consequente atuação mais precisa e equilibrada dos mesmos. Aponta-se ainda a importância de promover mais estudos com a arbitragem brasileira abordando o tema estratégias de coping e características de personalidade.

\section{Referências}

Allen, M. S., Greenlees, I., \& Jones, M. V. (2011). An investigation of the five-factor model of personality and coping behaviour in sport. Journal of Sports Sciences, 29(8), 841-850. https://doi.org/10.1080/02640414.2011.565064

Anshel, M. H. (2001). Qualitative validation of a model coping with acute stress in sport. Journal of Sport Behavior, 24, 223-246.

Antoniazzi, A. S., Dell'Aglio, D. D., \& Bandeira, D. R. (1998). O conceito de coping: uma revisão teórica. Estudos de Psicologia, 3(2), 273-294. https://doi.org/10.1590/S1413-294X1998000200006.

Bara, M. G. F., Ribeiro, L. C. S., \& Garcia, F. G. (2005). Comparação de características da personalidade entre atletas brasileiros de alto rendimento e indivíduos não atletas. Revista Brasileira de Medicina do Esporte, 11(2), 115-120. https:// doi.org/10.1590/S1517-86922005000200004

Boschilia, B., \& Marchi Júnior, W. (2008). Futebol, democracia e arbitragem: Algumas leituras figuracionistas. Jornal Esporte e Sociedade, 3(8), 1-18. Recuperado de http://www.uff.br/esportesociedade/pdf/es805.pdf

Brandão, M. R. F,, Serpa, S., Krebs, R., Araújo, D., \& Machado, A. A. (2011). El significado del arbitrar: percepción de jueces de fútbol profesional. Revista Psicología del Deporte, 20(2), 275-286. Recuperado de http://www.rpd-online.com/article/view/682/768

Carver, C. S., \& Connor-Smith, J. (2010). Personality and coping. Annual Review of Psychology, 61, 679-704. https://doi.org/10.1146/annurev.psych.093008.100352 
Cerqueira, M. S., Silva, A. I., \& Marins, J. C. B. (2011). Análise do modelo de avaliação física aplicado aos árbitros de futebol pela FIFA. Revista Brasileira de Medicina do Esporte, 17(6), 425-430. https://doi.org/10.1590/S151786922011000600012

Claudino, J., Costa, I., Simim, M., Teixeira, F., Silva, S., \& Pussieldi, G. (2012). A experiência pode ser um fator de alteração da percepção de estresse entre árbitros de futebol. EFDeportes.com - Revista Digital, 16(164). Recuperado de http://www.efdeportes.com/efd164/percepcao-de-estresse-entre-arbitros-de-futebol.htm

Cohen, S., Kamarck, T., \& Mermelstein, R. (1983). A global measure of perceived stress. Journal of Health and Social Behavior, 24(4), 385-396. Recuperado de http:// www.psy.cmu.edu/ scohen/globalmeas83.pdf

Coimbra, D. R., Bara, M. F., Andrade, A., \& Miranda, R.(2013). Habilidades psicológicas de coping em atletas brasileiros. Motricidade, 9(1), 95-106. https://doi.org/10.6063/motricidade.9(1).2467

Costa, V. T., Ferreira, R. M., Penna, E. M., Costa, I. T., Noce, F., \& Simim, M. A. M. (2010). Análise do estresse psíquico em árbitros de futebol. Revista Brasileira de Psicologia do Esporte, 3(2), 02-18. Recuperado de http:/ / pepsic.bvsalud.org/pdf/rbpe/v3n2/v3n2a02.pdf

Dalfovo, M. S., Lana, R. A., \& Silveira, A. (2008). Métodos quantitativos e qualitativos: Um resgate teórico. Revista Interdisciplinar Científica Aplicada, 2(3), 1-13. Recuperado dehttp://rica.unibes.com.br/index.php/rica/article/ view/243/234

De Rose Junior, D. (2002). A competição como fonte de estresse no esporte. Revista Brasileira de Ciência e Movimento, 10(4), 19-26. Recuperado dehttp://www.bibliotekevirtual.org/revistas/UCB/RBCM/v10n04/v10n04a02.pdf

Ferreira, R. A., \& Brandão, M. R. F. (2012). Árbitro brasileiro de futebol profissional: percepção do significado de arbitrar. Revista da Educação Física / UEM, 23(2), 229-238. https://doi.org/10.4025/reveducfis.v23i2.15235.

Ferreira, R. D. (2012). Árbitro de futebol profissional: motivos para início, permanência e disposição para o abandono da carreira (Dissertação de mestrado). Programa de Pós-Graduação em Educação Física, Universidade São Judas Tadeu, São Paulo, SP.

Gaudreau, P., \& Blondin, J.-P. (2002). Development of a questionnaire for the assessment of coping strategies employed by athletes in competitive sport settings. Psychology of Sport and Exercise, 3(1), 1-34. https://doi. org/10.1016/S1469-0292(01)00017-6

Gaudreau, P., Nicholls, A., \& Levy, A. R. (2010). The ups and downs of coping sports achievement: An episodic process analysis of within-person associations. Journal of Sport and Exercise Psychology, 32(3), 298-311. Recuperado de https://repository.edgehill.ac.uk/3048/1/U\%26Ds.pdf

Gimeno, F., Buceta, J. M., Lahoz, D., \& Sanz, G. (1998). Evaluación del proceso de toma de decisiones en el contexto del arbitraje deportivo: Propiedades psicométricas de la adaptación española del cuestionario DMQ II en árbitros de balonmano. Revista de Psicología del Deporte, 7(1), 249-260. Recuperado de http://www.rpd-online.com/ article/view/454/444

Gomes, S., Coimbra, D., Guillén, F., Miranda, R.,\& Bara Filho, M. (2007). Análise da produção científica em psicologia do esporte no Brasil e no exterior. Revista Iberoamericana de Psicología del Ejercicio y el Deporte, 2(1), 25-40. Recuperado de http://www.psicologiadeporte.ulpgc.es/docs/Analise_da_producao_cientifica_em_Psicologia_ do_Esporte_no\%20Brasil_e_no_exterior.pdf

González-Oya, J. L. (2006). Psicología aplicada al árbitro de fútbol: Características psicológicas y su entrenamiento. Sevilla: Wanceulen Editorial Deportiva.

González-Oya, J., \& Dosil Diáz, J. (2003). Compración de las habilidades psicológicas de árbitros de fútbol y baloncesto. Actas del Congreso Nacional de Psicología de la Actividad Física y el Deporte (pp. 258-264). León, Espanã, 9.

Gould, D., Ecklund, R. C., \& Jackson, S. A. (1993). Coping strategies used by U.S. Olympic Wrestlers. Research Quarterly for Exercise and Sport, 64(1), 83-93. https://doi.org/10.1080/02701367.1993.10608782

Hardy, L., Jones, G., \& Gould, D. (1996). Understanding psychological preparation for sport: Theory and practice of elite performers. Chichester: Wiley.

Helsen, W., \& Bultynck, J. B. (2004). Physical and perceptual-cognitive demands of top-class refereeing in association football. Journal of Sports Sciences, 22(2), 179-189. https://doi.org/10.1080/02640410310001641502

Kerdijk, C., Kamp, J., \& Polman, R. (2016). The Influence of the social environment context in stress and coping in sport. Frontiers in Psychology, 7, 875. https://doi.org/10.3389/fpsyg.2016.00875 
Kowalski, K. C., \& Crocker, P. R. (2001). Development and validation of the Coping Function Questionnaire for adolescents in sport. Journal of Sport \& Exercise Psychology, 23(2), 136-155. https://doi.org/10.1123/jsep.23.2.136

Krohne, H.W. (2001) Stress and coping theories. The International Encyclopedia of the Social and Behavioral Sciences, 22, 15163-15170. https://doi.org/10.1016/B0-08-043076-7/03817-1

Lazarus, R. S. (1964). A laboratory approach to the dynamics of psychological stress. American Psychologist, 19(6), 400-411. http://dx.doi.org/10.1037/h0041245

Lazarus, R. S. (2000). How emotions influence in competitive sports. The Sport Psychologist, 14, 229-252. Recuperado de http://tonypickering.com/documents/Lazarus_2000.pdf

Lazarus, R. S., \& Folkman, S. (1984). Stress appraisal, and coping. New York, NY: Springer.

Lopez, A. V., \& Santelices, O. Y. S. (2011). Personality characteristics of elite table tennis athletes of the Philippines: Basis for a proposed recruitment program. ITTF Sports Science Congress, Rotterdam, The Netherlands. 12. Recuperado de http://www.ittf.com/ittf_science/SSCenter/docs/01-05\%20Lopez.pdf

Luft, C. B., Sanches, S. O., Mazo, G. Z., \& Andrade, A. (2007). Versão brasileira da Escala de Estresse Percebido: Tradução e validação para idosos. Revista Saúde Pública, 41(4), 606-15. https://doi.org/10.1590/S003489102007000400015

Malinauskas, R., Dumciene, A., Mankus, G. \& Vekckunas, T. (2014). Personality traits and exercise capacity in male athletes and non athletes. Perceptual \& Motor Skills: Motor Skills \& Ergonomics, 24(4), 445-452. https://doi.org/10.2466/29.25.PMS.118k13w1

Mimura, C., \& Griffiths, P. (2004). A Japanese version of the perceived stress scale: Translation and preliminary test. International Journal of Nursery Studies, 41(4), 379-385. https:// doi.org/10.1016/j.ijnurstu.2003.10.009

Mimura, C., \& Griffiths, P. (2008). A Japanese version of the Perceived Stress Scale: cross-cultural translation and equivalence assessment. BMC Psychiatry, 8, 85. https://doi.org/10.1186/1471-244X-8-85

Nicholls, A. R., Holt, N. L., Polman, R. C., \& Bloomfield, J. (2006). Stressors, coping and coping effectiveness among Professional rugby union players. The Sport Psychologist, 20(3), 314-329. https://doi.org/10.1123/tsp.20.3.314

Olsen, L. R., Mortensen, E. L., \& Bech, P. (2004). Prevalence of major depression and stress indicators in the Danish general population. Acta PsychiatricaScandinavica, 109(2), 96-103.

Örücü, M. C., \& Demir, A. (2008). Psychometric evaluation of perceived stress scale for Turkish university students. Stress and Health, 25(1), 103-109. https://doi.org/10.1002/smi.1218

Paes, M. R., Fernandez, R., \& Silva, A. I. (2011). Injuries to football (soccer) referees during matches, training and physical tests. International Sport Medicine Journal, 12(2), 78-84.

Pais-Ribeiro, J. L. \& Marques, T. (2009). A avaliação do estresse: a propósito de um estudo de adaptação da escala de percepção de estresse. Psicologia, Saúde \& Doenças, 10(2), 237-248. Recuperado dehttp://www.scielo.mec.pt/ scielo.php?script =sci_arttext\&pid=S1645-00862009000200008\&lng =pt\&nrm=iso\&tlng=pt

Patiño, O., \& Cañadas, M. (2015). Análisis de la figura del árbitro deportivo y su intervención en el proceso de formación deportiva. Revista Pedagógica Adal, 18(30), 25-32. Recuperado de https://issuu.com/apefadal/docs/ revista_30_final

Pereira, N. F., Santos, R. G. M., \& Cillo, E. N. P. (2007). Arbitragem no futebol de campo: Estresse como produto desse controle coercitivo. Revista Brasileira de Psicologia do Esporte, 1(1), 01-11. Recuperado de http://pepsic.bvsalud. org/scielo.php?script=sci_arttext\&pid=S1981-91452007000100004

Quintero, E. G. (2007). Reseña de "Psicología aplicada al árbitro de fútbol. Características psicológicas y su entrenamiento" de Jacinto González Oya [Resenha de Psicología aplicada al árbitro de fútbol: Características psicológicas y su entrenamiento, de J. G. Oya]. Revista Iberoamericana de Psicología del Ejercicio y el Deporte, 2(1), 127-130. Recuperado de http://www.redalyc.org/articulo.oa?id=311126252010

Ramírez, M. T., \& Hernández, R. L. (2007). Factor structure of the Perceived Stress Scale (PSS) in a sample from Mexico. The Spanish Journal of Psychology, 10(1), 199-206. https://doi.org/10.1017/S1138741600006466

Ramos, F. P., Enumo, S. R. F., \& Paula, M. P. (2015). Teoria Motivacional do Coping: uma proposta desenvolvimentista de análise do enfrentamento do estresse. Estudos de Psicologia Campinas, 32(2), 269-279. https://doi. org/10.1590/0103-166X2015000200011 
Reilly, T., \& Warren, G. (2006). Special populations: The referee and assistant referee. Journal of Sports Sciences, 24(7), 795-801. https://doi.org/10.1080/02640410500483089

Reis, R. S., Hino, A. A., \& Añez, C. R. (2010). Perceived stress scale: reliability and validity study in Brazil. Journal of Health Psychology, 15(1), 107-114. https://doi.org/10.1177/1359105309346343

Remor, E. (2006). Psychometric properties of an european Spanish version of the Perceived Stress Scale (PSS). The Spanish Journal of Psychology, 9(1), 86-93. Recuperado de http://www.psy.cmu.edu/ scohen/Remor_2006_article_EurSpanPSS.pdf

Ribeiro, J. P. \& Marques, T. (2009). A avaliação do stresse: A propósito de um estudo de adaptação da Escala de Percepção de Stresse. Psicologia, Saúde \& Doenças, 10(2), 237-248. Recuperado de http://www.scielo.mec.pt/pdf/ psd/v10n2/v10n2a07.pdf

Skinner, E. A. (1999). Action regulation, coping and development. In J. B. Brandtstädter \& R. M. Lerner (Eds.), Action and self-development (pp.465-503). Thousand Oaks, CA: Sage.

Silva, A.S. (2004). Construção e validação de um instrumento para medir o nível de estresse dos árbitros dos jogos esportivos coletivos (Dissertação de mestrado). Escola de Educação Física, Fisioterapia e Terapia Ocupacional, Universidade Federal de Minas Gerais, Belo Horizonte, Minas Gerais.

Vaillant, G. E. (1994). Ego mechanisms of defense and personality psychopathology. Journal of Abnormal Psycho$\log y, 103(1), 44-50$.

Velho, N. M., \& Fialho, A. F. P. (2015). Competências básicas na formação de árbitros de futebol. EFDeportes.com, 20(208). Recuperado de http://www.efdeportes.com/efd208/competencias-basicas-de-arbitros-de-futebol.htm

\section{Alessandra Carla Peixoto Monteiro}

Psicóloga da Federação Mineira de Futebol. Mestre em Psicologia pela Universidade Federal de Minas Gerais (UFMG), Belo Horizonte - MG, Brasil.

E-mail: esportivamente@globo.com

\section{Mariana Verdolin Guilherme Froeseler}

Psicóloga. Mestre eDoutoranda emPsicologiapelaUniversidadeFederal deMinas Gerais (UFMG), Belo Horizonte-MG, Brasil.

E-mail: marianaverdolingf@gmail.com

\section{Marcela Mansur-Alves}

Doutora em Neurociências pela Universidade Federal de Minas Gerais (UFMG), Belo Horizonte - MG, Brasil. Professora Adjunta do Programa de Pós-Graduação em Psicologia: Cognição e Comportamento (UFMG).

E-mail: marcelamansura@gmail.com

Endereço para envio de correspondência:

Faculdade de Filosofia e Ciências Humanas - Departamento de Psicologia (sala 4080).

Av. Pres. Antônio Carlos, 6.627, campus Pampulha, CEP: 31270-901. Belo Horizonte - MG. Brasil.

Recebido 11/09/2016

Reformulado 20/06/2017

Aprovado 24/07/2017

Received 09/11/2016

Reformulated 06/20/2017

Approved 07/24/2017 
Recibido 11/09/2016

Reformulado 20/06/2017

Aceptado 24/07/2017

Como citar: Froeseler, M. V. G., Monteiro, A. C. P., \& Mansur-Alves, M. (2018). Estresse e coping de árbitros de futebolnotestefísicoFIFA. Psicologia:CiênciaeProfissão,38(1),102-115.https://doi.org/10.1590/1982-3703003492016

How to cite: Froeseler, M. V. G., Monteiro, A. C. P., \& Mansur-Alves, M. (2018). Stress and coping of soccer referees in the FIFA physical test. Psicologia:Ciência e Profissão, 38(1), 102-115. https://doi.org/10.1590/1982-3703003492016

Cómo citar: Froeseler, M. V. G., Monteiro, A. C. P., \& Mansur-Alves, M. (2018). Estrés y coping de árbitros de fútbol en la prueba física FIFA. Psicologia:Ciência e Profissão, 38(1), 102-115. https://doi.org/10.1590/1982-3703003492016 